\title{
Les démonstrations de gestes endoscopiques en direct : intérêt pédagogique et sécurité des patients
}

\section{Live demonstrations of endoscopic procedures: educational benefits and patient safety}

\author{
C. Cellier $\cdot$ B. Richard-Molard $\cdot$ C. Boustiere $\cdot$ J.-M. Canard $\cdot$ J.-P. Arpurt $\cdot$ D. Bernardini $\cdot$ P. Bulois $\cdot$ S. Chaussade $\cdot$ \\ D. Heresbach · I. Joly · J. Lapuelle · R. Laugier · G. Lesur · P. Pienkowski · T. Ponchon · B. Pujol · \\ M. Robaszkiewicz $\cdot$ R. Systchenko • pour la Société française d'endoscopie digestive
}

(C) Springer-Verlag France 2012

La dernière édition de Vidéo-Digest vient de se tenir à Paris et Avignon et a été un succès appréciable avec près de 1000 participants. C'est l'occasion pour le CA de la SFED de répondre au récent billet d'humeur de Philippe Levy.

Ce récent «billet d'humeur » publié dans le journal a comparé les démonstrations en direct d'endoscopie à une corrida et a suggéré une possible perte de chance pour les patients. Il n'échappe à personne que ce billet d'humeur,

\footnotetext{
C. Cellier $(\bowtie)$

Hôpital européen Georges-Pompidou, 20, rue Leblanc, F-75015 Paris, France

e-mail : christophe.cellier@egp.ap-hop-paris.fr

B. Richard-Molard

15, rue Claude-Boucher, F-33300 Bordeaux, France

C. Boustiere

Service d'endoscopie digestive, hôpital privé Saint-Joseph, boulevard de Louvain, F-13285 Marseille cedex 08, France

J.-M. Canard

96, boulevard du Montparnasse, F-75014 Paris, France

J.-P. Arpurt

Centre hospitalier, 305, rue Raoul-Follereau, F-84902 Avignon, France

D. Bernardini

Hôpital Font Pré, service d'hépatogastroentérologie, 1206 avenue Colonel Picot, F-83056 Toulon cedex

P. Bulois

Hôpital Saint-Philibert, rue du Grand-But, BP 249, F-59462

Lomme cedex, France

S. Chaussade

Hôpital Cochin, AP-HP, 27, rue du Faubourg-Saint-Jacques, F-

75679 Paris cedex 14, France

D. Heresbach

Unité d'endoscopie digestive, centre hospitalier de Cannes, 15, avenue des Broussailles, CS 50008, F-06414 Cannes, France
}

placé en face de l'annonce de Vidéo-Digest, vise en particulier cette réunion organisée par la SFED. Ce billet d'humeur amène de la part de la SFED la réponse suivante.

Sur la forme, il est vraiment déplacé de comparer les démonstrations en direct d'endoscopie à une corrida, avec tout ce que cela sous-entend, en particulier concernant la mort du taureau. Sur le fond, la SFED, qui organise VidéoDigest depuis 20 ans, n'a pas attendu ce billet d'humeur pour

I. Joly

9, boulevard Clémenceau, F-22000 Saint-Brieuc, France

J. Lapuelle

Clinique Saint-Jean-Languedoc, 20, route de Revel, F-31400

Toulouse, France

R. Laugier

Hôpital La Timone, AP-HM, 264, rue Saint-Pierre, F-13385

Marseille cedex 05, France

G. Lesur

Hôpital Ambroise-Paré, 9, avenue Charles-de-Gaulle, F-92104

Boulogne cedex, France

P. Pienkowski

Clinique du Pont-de-Chaume, F-82017 Montauban, France

T. Ponchon

Hôpital Édouard-Herriot, pavillon H, place d'Arsonval, F-69347

Lyon cedex 03, France

B. Pujol

40, cours Gambetta, F-69007 Lyon

M. Robaszkiewicz

Hôpital de la Cavale-Blanche, boulevard Tanguy-Prigent, F-

29609 Brest cedex, France

R. Systchenko

5 , avenue de Verdun, F-69540 Irigny 
s'interroger et se positionner sur la valeur pédagogique et sur le caractère éthique d'une telle réunion.

Au-delà de la polémique, la question posée est la suivante : peut-on remplacer les démonstrations en direct par des démonstrations enregistrées ? Ces deux types de démonstration sont complémentaires. Les démonstrations en direct se distinguent des démonstrations enregistrées sur trois points :

- elles ont un caractère prospectif en montrant sans biais de sélection les décisions prises par l'opérateur. Alors que les démonstrations enregistrées ont un caractère rétrospectif, c'est-à-dire que l'opérateur montre ce qu'il veut bien montrer ;

- les démonstrations en direct montrent non seulement la technique, comme les démonstrations enregistrées, mais elles enseignent aussi la maîtrise en direct de la gestuelle et de la situation. L'enseignant doit non seulement être un bon technicien, mais il doit aussi assurer le rôle de stratège et de modèle, et seul le direct peut faire passer correctement ces aspects de l'enseignement, au mieux pour une petite audience dans une salle d'endoscopie, ou sinon par retransmission pour une large audience ;

- les démonstrations en direct permettent le mieux d'apprécier le degré de difficulté d'un geste. Et l'audience vient aussi rechercher cette information.

Bien entendu, ces démonstrations ne doivent pas se faire au prix d'une perte de chance pour le patient. L'ESGE comme l'ASGE ont défini des critères qualité pour la réalisation de live [1], et les organisateurs d'un des plus importants cours en « live » européens n'ont pas observé d'augmentation de la morbidité des gestes lors de ce « live » [2]. La SFED suit ces critères de qualité, et au dire des experts internationaux, Vidéo-Digest est la démonstration en directe d'endoscopie digestive la plus exigeante sur le plan de l'éthique et de la qualité des soins. Pour information, le déroulé de Vidéo-Digest est détaillé en annexe A (sauf pour ce qui concerne la partie logistique) et nous vous invitons à le lire. Les points principaux sont : un cahier des charges pour les centres, un encadrement de la préparation pendant 18 mois par un responsable de la SFED, le consentement des patients à la vidéotransmission, une journée préparatoire consacrée à débattre des indications et du matériel, une séance « retour sur le direct». Contrairement à ce que le laisse entendre le billet d'humeur, Vidéo-Digest offre aux patients des conditions optimales de réalisation de leur geste, conditions qui ne sont d'ailleurs pas toujours retrouvées en pratique quotidienne : double information du patient (par celui qui a en charge le patient et par l'opérateur), discussion collégiale du geste et des indications, matériel le plus performant, opérateur expert du geste à réaliser.

Nous sommes ouverts à toute discussion et critique sur le fond concernant les modalités des formations que la SFED met en œuvre. Nous souhaitons que ces critiques se fassent sous une forme dépassionnée et en toute connaissance de cause, tenant compte que la SFED a tout fait depuis des années pour que Vidéo-Digest soit un modèle de démonstrations en direct et un modèle tout court de la pratique de l'endoscopie.

\section{Annexe A. Échéancier de Vidéo-Digest : phases médicales}

j- 18 mois : Choix du centre et désignation du responsable SFED. Le centre doit appliquer un cahier des charges précis (moyens humains et matériels, ressources techniques, choix des gestes endoscopiques, information des patients, responsabilité des établissements, lieux d'hospitalisation, formation et information du personnel infirmier, information et implication des services partenaires : anesthésie, anatomopathologie, radiologie). En parallèle, le comité d'organisation de Vidéo-Digest désigne un hépatogastroentérologue (responsable SFED pour la manifestation) pour :

- assister le responsable médical local dans l'organisation ;

- comodérer avec le responsable médical local la journée de finalisation ;

- assurer le déroulement du programme le jour du direct en collaboration avec le responsable local.

Ce responsable SFED va visiter le centre d'endoscopie en avance et va assister aux réunions avec les structures administratives et techniques locales.

j - 10 mois : Réunion (responsable de la SFED, responsables locaux médicaux) pour le choix des gestes endoscopiques à montrer et le choix des experts en fonction des gestes décidés.

j- 5 mois : Réunion (responsables de la SFED, responsables locaux médicaux, fabricants) pour confirmation sur les gestes et les opérateurs.

Établissement de la liste de matériel dont a besoin transitoirement l'unité pour compléter le parc : endoscopes, consoles...

j - 2 mois - 1 mois : Arrivée dans le centre du matériel complémentaire, afin que le personnel paramédical du centre puisse se familiariser à la mise en œuvre de ce matériel (désinfection en particulier). Organisation de workshops internes de complément de formation sur le matériel.

j - 2 semaines - 6 mois : Information aux patients et responsabilité des experts

Les patients reçoivent non seulement l'information habituelle sur le geste endoscopique, mais ils donnent leur consentement signé à ce que le geste soit réalisé dans le cadre de Vidéo-Digest, après information détaillée sur les particularités liées à la vidéotransmission (opérateur extérieur, présence de caméras, auditoire...). La liste des experts 
est transmise aux services administratifs de l'établissement afin que celui-ci donne son accord et prenne une assurance couvrant la pratique des experts.

j - 1 - matin : Toute la journée, la veille du direct est consacrée à la finalisation du programme : Les opérateurs et les organisateurs locaux (y compris les anesthésistes) sont présents le matin pour discuter de tous les cas, un par un, avec toute la documentation nécessaire. Certains cas sont écartés, des indications sont modifiées... Ce qui veut dire que le geste à réaliser pour tel ou tel cas est bien défini à l'avance. Le principe est que lors du direct, la discussion des indications soit minimale (sauf si un nouvel élément est révélé par le geste lui-même), de façon à ce que le patient n'ait pas à pâtir d'une discussion non contrôlée et que l'opérateur puisse réaliser ce qu'il pense devoir réaliser sans pression. L'audience va donc peu intervenir sur les indications, mais va pouvoir intervenir sur les aspects techniques. $\mathrm{La}$ décision de faire tel ou tel geste incombe donc à l'opérateur et au correspondant local du patient. Pour que les indications soient néanmoins discutées avec la salle, il est organisé le lendemain du direct une session entre les opérateurs et l'audience (« retour sur le direct») pour que celle-ci revienne sur les indications et pose toutes les questions qu'elle souhaite à ce sujet.

j-1 - après-midi : Le matériel est définitivement choisi par les opérateurs en fonction des cas à réaliser et de leur expertise, en présence des fabricants. À la fin de la journée (ou le matin pour les gestes les plus simples en ambulatoire), chaque opérateur va voir les patients dont il devra s'occuper, dans leur chambre, pour se présenter et leur donner un complément d'information. j0 - live : Le responsable de la SFED gère le déroulement du programme, les responsables locaux gèrent le suivi médical des patients, de façon à ce que les responsables locaux soient totalement disponibles pour les patients, avant, pendant et après, et n'aient pas à se préoccuper de la logistique. Pour chaque cas, deux experts sont présents, l'un qui est l'opérateur et l'autre qui est l'interface entre l'opérateur et l'audience. Selon les cas, selon les opérateurs, le second expert intervient pour aider l'opérateur dans sa prise de décision, pour filtrer les questions et si nécessaire pour soulager l'opérateur d'une quelconque pression. Les cas sont enregistrés en partie pour être discutés le lendemain. Il arrive que des cas ne puissent pas être montrés dans les périodes de direct. Les patients sont d'ailleurs informés de cette possibilité. C'est une situation prévue, et des membres de l'équipe locale sont disponibles pour finir le programme le soir ou le lendemain et pour prendre en charge les éventuelles complications.

j plus 1 : Retour sur le direct (cf. plus haut).

\section{Bibliographie}

1. Devière J, Ponchon T, Beilenhoff U, Neuhaus H, Costamagna G, Schmit A, et al. Recommendations of the ESGE Worshop on Ethical-Legal issues concerning live demonstration in digestive endoscopy. Endoscopy 2003;35:765-7.

2. Lévy P. Corrida et démonstation live en endoscopie. Hepato Gastro 2012;19:469-70

3. Schmit A, Lazaraki G, Hittelet A, Cremer M, Le Moine O, Devière J. Complications of endoscopic retrograde cholangiopancreatography during live endoscopy workshop demonstrations. Endoscopy 2005;37:695-9. 